\title{
ASSESSMENT OF THE USABILITY OF LATVIA'S OPEN DATA PORTAL OR HOW CLOSE ARE WE TO GAINING BENEFITS FROM OPEN DATA
}

\author{
Anastasija Nikiforova \\ Faculty of Computing, University of Latvia, Rainis Boulevard 19. Riga, LV-1586, Latvia
}

\begin{abstract}
Nowadays, more and more countries are launching their own open data portals, seeking to provide their citizens with open data in a form that is useful and suitable for the original purpose of the open data, and Latvia is not an exception. Despite the fact that the Latvian open data portal was launched only in 2017 , it is considered to be a fast-tracker. However, despite the overall high evaluations, critical voices, and many discussions about whether the Latvia's open data portal is of sufficient quality to be appeared. Therefore, while previous studies deal with quality of open data, this study focuses on the analysis of the Latvian open data portal and aims to find the key challenges that may have a negative impact on user experience. The paper assesses the current situation and recommends corrective actions, highlighting the aspects to be considered when developing and improving open data portals.
\end{abstract}

\section{KEYWORDS}

Open Data, Open Data Government, Open Data Portal, Portal Quality, Usability

\section{INTRODUCTION}

Nowadays, open data and in particular open government data (OGD) are the topics underpinning the recent governance, policy and process innovations around the world. However, open data itself does not play an important role since value is generated by the use of data (Faini et al., 2016). According to (Janssen et al., 2012), one of the primary objectives in the case of OGD is to accompany publicized data by an infrastructure - open data portal - capable of processing data in an easy-to-use manner to reduce the user threshold, thus maximising their re-use to a wide audience (Attard et al., 2015). As a result, more and more governments are establishing open data portals where different types of data are published by different data providers and Latvia is not an exception. However, despite many countries develop and launch their own OGD portals, they received a great deal of criticism from both, society, by which is meant users who may not have IT knowledge, and technical experts. Latvia is not an exception and one of the most important reasons to initiate a study were the critical voices appeared in Latvia, even though according to different international assessments, Latvia's Open Data Portal is considered as qualitative. Many discussions about (1) whether our Open Data Portal is of adequate quality? and (2) what are the main challenges to be addressed? took place, and this study addresses these issues. This was also discussed at the $13^{\text {th }}$ annual conference of the Latvian Open Technology Association "Data Driven Nation", in which the author was an invited speaker.

Since the Latvian open data portal is relatively new, it is not included in other studies, while other portals of our neighbours (Lithuania, Estonia, Finland) have been investigated. Therefore, this study is unique to Latvia and has a high national value. However, in order to bring added value at the international level, this paper provides brief comparison with other open data portals, highlighting the aspects to be taken into account when developing and improving open data portals.

To sum up, the aim of this paper is to explore Latvia's Open Data portal, by evaluating it from different perspectives. As for the methods used, the number of existing methods was studied, selecting (Máchová et al., 2018) usability evaluation framework, supplying it with the author's requirements that the portal must satisfy which were formulated on the basis of experience with the portal. 
The paper addresses the following issues: a brief overview of the related studies (Section 2), an overview of Latvia's open data portal (Section 3), an analysis of Latvia's open data portal, highlighting its main challenges (Section 4) and conclusions (Section 5).

\section{RELATED WORKS}

The relevance and popularity of [open] data portals and OGD topics are also reflected in the number of studies. According to leading libraries collecting researches, namely, ACM Digital Library, Science Direct, Springer Link, Web of Science, IEEE Xplore Digital Library, the total number of studies on open data portals and OGD published in recent years is less than $29 \%$ of the studies dedicated to open data (Figure 1). For Google Scholar, the number of open data studies exceeds the number of open data portal and OGD studies nearly 15 times - the ratio to total number of open data studies is $\sim 6.25 \%$.

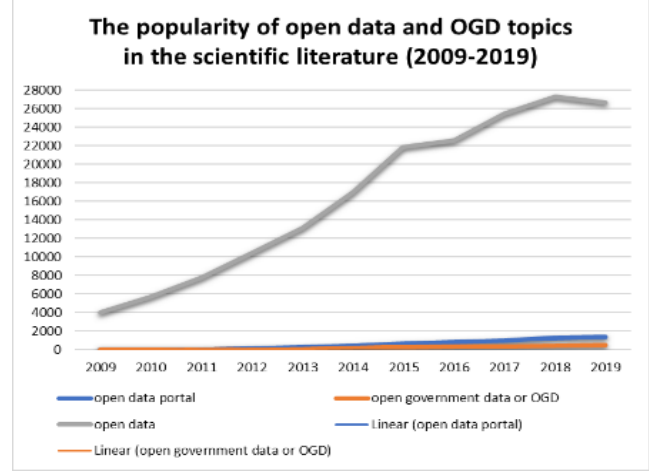

Figure 1. The popularity of open data and OGD topics

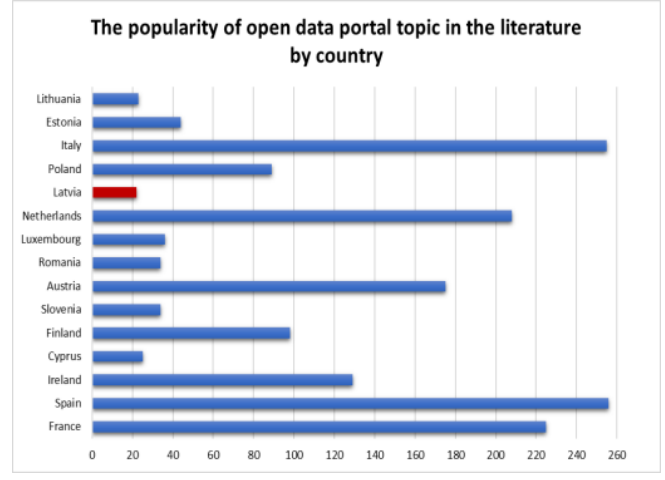

Figure 2. The popularity of open data and OGD topics

According to Figure 2, which shows how many studies were conducted by country on an open data portal, only a few studies were devoted to Latvia in the way of open data portals. Compared to the leading countries, the topic of Latvia's open data portal is covered nearly 12 times rarely $(1.33 \%$ of all studies, while for the leading countries this ratio exceeds $15 \%$ ). In most cases, the Latvian open data portal was mentioned in only one or a few sentences. Closest to the presented study - the analysis of the open data portal, was carried out in 2014 (Bojars et al., 2014), however, it was dedicated to another portal that no longer exists. For the most popular countries covered in scientific literature, they are Spain, France, Italy, Netherlands, Austria, Ireland and Finland, among which only Italy has a lower score of the state of the open data portal (according to European Data Portal). Therefore, it can be assumed that the popularity of this topic in scientific literature may sometimes (but not necessarily) be linked to the state of the open data portals, as the number of studies demonstrates the public's (at least the scientific part of society) interest in the subject.

For more specific studies, a number of benchmarks and indexes were recently introduced assessing the state of OGD - the Global Open Data Index, EU Open Data maturity indicators, Open Data Barometer, etc.

The Global Open Data Index follows the state of the OGD, that is, which countries publish the data in the right way and in timely manner. Currently, 94 countries are included in the index of 15 key datasets per country, and only $11 \%$ of the data set entries were open according to their open definition. Since data are currently available only for 2016 (because the evaluation process is quite complex), their finding may be considered outdated. This is also the case for Latvia, since many improvements in the state of open data [related to the launch of the national open data portal] took place.

Another example is the Open Data Barometer, which aims to provide a snapshot of OGD practices. The Open Data Barometer is focused on open data readiness, implementation, and emerging impacts. The most urgent, the $4^{\text {th }}$ edition assessed these aspects for a sample of 30 countries. However, this assessment may also be considered to be outdated as the data provided reflect the state of OGDs in 2017. Moreover, since 113 countries were covered in 2016, it can be assumed that even for 2017 there was only a partial report.

The European Data Portal carries out an evaluation of national open data portals, assessing 4 key aspects, namely policy, portal, impact and quality. This assessment appears to be one of the most up-to-date assessments covering set of open data portals, as they have published results for 2019. According to their 
analysis, impact is currently the weakest aspect of OGDs, following by quality (partially addressed in (Nikiforova, 2020a)) and portal. As for the portal category, they focus on evaluation of portal functions that allow both versed and less versed users to access open data through the national portal and features that encourage interaction between publishers and re-users.

According to (Attard et al., 2015) and literature review performed by the author (partly covered in (Nikiforova, 2020b)), many studies cover their own national OGD initiatives, where for most studies, a lack of a common methodology that would allow comparisons between studies is observed.

Since the OGD may be used to create a new public service, one of the tasks to be performed is to get an overview of the available OGD datasets. Therefore, this study analyses not only a portal but also its datasets. For the approach to be used in this study, as a result of the analysis of existing studies, the preference was given to the (Máchová et al., 2018) methodology. This study proposes to evaluate open data portal using 3 key aspects - open data specification, open dataset feedback, open dataset request, divided into 14 sub-aspects (see subsection 4.2). This methodology was found to be appropriate for a number of reasons: (a) it is relatively new (less than 2 years old), (b) cited, meaning that it is accepted by the public/ researchers, (c) complies with the principles used by other researchers as the methodology is similar with (Attard et al., 2015), (Zuiderwijk et al., 2015), (d) easy to replicate; (e) considers a user perspective. It is also should be mentioned that despite the potential impact of the open data portals web interface on portal usability, this paper does not address it, leaving it for other studies.

\section{LATVIA'S OPEN DATA PORTAL: GENERAL REPORT}

Latvia's open data portal (data.gov.lv) was launched in 2017. At the time of its launch it collected only 33 datasets from 13 data publishers, however, in June 2020 the total number of datasets exceeds 395 from 74 data publishers, which is the highest among neighbours from the Baltic states.

As regards its development, Latvia is one of 79 countries participating in the Open Government Partnership. According to Open Data Maturity report 2019 (Blank, 2019), that measures the maturity of open data across Europe, Latvia belongs to fast-trackers (among beginners, followers, fast-trackers, trend-setters) and has the highest level of maturity of open data compared to the Baltic States and Scandinavian countries. Furthermore, in recent years, the open data maturity of Latvia's open data [portal] has been steadily improving (Nikiforova, 2019). The maturity of Latvia's open data portal is assessed as the $11^{\text {th }}$ among 32. In terms of general evolution, Latvia demonstrates the most impressive results, improving its position from the first appearance in this rating by getting from position 30 to 11 (Table 1).

Table 1. Maturity of portals

\begin{tabular}{|c|c|c|c|c|c|}
\hline \# & Country/year & 2019 & 2018 & 2017 & 2016 \\
\hline 1 & France & $\mathbf{1} \uparrow+4$ & $\mathbf{5} \uparrow+3$ & $8 \downarrow-5$ & 3 \\
\hline 2 & Spain & $\mathbf{2} \uparrow+6$ & $8 \downarrow-3$ & $5 \downarrow-1$ & 4 \\
\hline 3 & Ireland & 3 & 3 & $3 \uparrow+7$ & 10 \\
\hline ... & ... & $\ldots$ & $\ldots$ & $\ldots$ & ... \\
\hline 11 & Latvia & $\mathbf{1 1} \uparrow+8$ & $\mathbf{1 9} \uparrow+2$ & $\mathbf{2 1} \uparrow+9$ & 30 \\
\hline ... & $\ldots$ & $\ldots$ & $\ldots$ & $\ldots$ & $\ldots$ \\
\hline
\end{tabular}

Moreover, Latvia is one of 4 countries (Ireland, Latvia, Italy, Malta) which improves its positions from year to year (for more details see (Nikiforova, 2020b)).

\section{ANALYSIS OF LATVIA'S OPEN DATA PORTAL}

The analysis of Latvia's open data portal was carried out in 3 stages: (1) a questionnaire of Latvian citizens; (2) the methodology applied to the portal by 35 users with IT background; (3) additional aspects not covered 
in the applied methodology (or not detailed enough), that were defined by the author of this paper as the user of this portal based on observations, analysed by inspecting every data set (out of 395).

\subsection{Survey on the Latvian Open Data Portal}

As critical voices appear in Latvia more and more frequently, the first step in the analysis of the Latvia's Open Data Portal was a questionnaire. 105 voluntary participants were involved representing three groups: (a) users with IT background, (b) without IT background, divided into two additional groups: undergraduate and graduated. A list of simple questions was asked, in which the most important in the scope of this paper, are (1) whether the participant knows that Latvia has its own national open data portal?, if "yes" (2) how hel she assesses it (2.1.) from a "usability" perspective, (2.2.) from the quality of the data published in it.

The results demonstrate that only $53 \%$ of respondents are aware of the existence of Latvia's open data portal and $13.5 \%$ suppose that this exists, however, are not sure. However, $74.3 \%$ of those, who know about the existence of the portal, have not used it, $18 \%$ used it only once, and only $7.7 \%$ use it in a daily manner. An interesting point is that the number of non-IT experts who are aware of the existence of Latvia's open data portal is higher than the number of users with IT background by $13 \%$. However, this trend is not valid in the case of its use, as only $7.7 \%$ of non-IT users use this portal regularly, while for IT-users this ratio is $12 \%$.

As for the assessment, which is a subjective opinion of each respondent, the overall usability of the open data portal was assessed by 6.3 points out of 10 , while the quality of published data -6.2 out of 10 . The assessment received from IT users was lower by 1 point in the case of usability and 0.5 point in the case of quality. These results demonstrate that despite the high results according to international assessment systems, Latvian users are not satisfied with this portal in the highest way. This proves the importance of this study in determining aspects assessed by users as not high enough.

As for the next stage of the study, only users with IT background participated in it, that corresponds to the experiment design of (Máchová et al., 2018).

\subsection{Results of the Application of the Methodology}

As in (Máchová et al., 2018), a three-level Likert scale (fulfilled - 3, partially fulfilled - 2, and unfulfilled - 1), that belongs to the acceptability tasks, was used to measure the usability of open data portal. Latvia's open data portal was assessed by 35 participants selected on the voluntary basis using in-group (between subject) observational method to reduce the potential subjectivity of the results, which cannot be achieved if the total number of participants is less than 30. Table 2 summarises the results of the analysis, where a total score can vary from 14 to 42 points. Latvia was scored with 31.5 out of $42(62.5 \%)$ while leading countries got between 35.6 and 38.2 points (Nikiforova, 2020b).

Table 2. Assessment of Latvia's open data portal

\begin{tabular}{clc}
\hline Category & \multicolumn{1}{c}{ Aspect } & Points (1 to 3 points) \\
\hline & a) Description of dataset & $\mathbf{2 , 0 7}$ \\
& b) Publisher of dataset & $\mathbf{2 , 7 4}$ \\
Open dataset & c) Thematic categories and tags & $\mathbf{2 , 3 6}$ \\
specification & d) Release date and up to date & $\mathbf{2 , 9}$ \\
& e) Machine-readable formats & $\mathbf{2 , 1}$ \\
& f) Open data licence & $\mathbf{3}$ \\
& g) Visualization and statistics & $\mathbf{1 , 2 6}$ \\
Open dataset & a) Documentation and tutorials & $\mathbf{1 , 9 8}$ \\
feedback & b) Forum and contact form & $\mathbf{2 , 0 2}$ \\
& c) User rating and comments & $\mathbf{1 , 7 6}$ \\
Open dataset & d) Social media and sharing & $\mathbf{2 , 5 2}$ \\
request & a) Request form & $\mathbf{2 , 8 3}$ \\
& b) List of requests & $\mathbf{1 , 8 8}$ \\
\hline 1 point - not fulfilled, 2 points - partially fulfilled, 3 points - fulfilled & $\mathbf{2 , 0 5}$ \\
n- weak/ low result (under 2 points out of 3) requiring improvements &
\end{tabular}


Let's briefly discuss the main results and challenges identified. For the first category, namely "open data set specification", while the portal provides an opportunity to describe the data set to be published, data publishers often do not provide any or sufficiently detailed descriptions, and in many cases only the name of data set is recopied as a description. As regards the "publisher of dataset", this information is mandatory for the publication of open data in Latvia's open data portal, therefore, this requirement is always fulfilled. Aspects such as "machine-readable format" and "open data licence" are one of those that ensured Latvian open data portal with such a high position in international ratings as all datasets meet these requirements. For the licence, all data sets correspond to CC0 1.0. This is also in line with (Máchová et al., 2018), where all the assessed portals were found to meet this requirement because open data license is today the key part of each portal. However, only a few years ago it was a major challenge for the open data portals, and, even now, in 2020 , there are portals that either do not provide information regarding license, or use multiple licenses, where some of them assign limitations on data sets to be used, for instance, Germany, Greece, Hungary, Czech Republic (see (Nikiforova, 2020b)). As for "thematic categories and tags", despite 14 different categories exist, by the beginning of June 2020 there were 102 datasets not belonging to any category. It is not related to poor portal design, but the publisher did not specify a category that should be assigned to the specific data set. For instance, one of the data sets not belonging to any category, namely "Distribution of scientific articles by genders and scientific disciplines in Web of Science 2015-2019" could be linked to the "Education" category etc. However, as a result, this makes it impossible to find datasets that are not assigned to any categories if a user studies datasets by category. In such a case, the two recommended solutions: (a) make the choice of categories mandatory for data publishers; (b) include the category "other" or "uncategorized", as in the case of Bulgaria. Of course, one of the best options would be involving a mechanism that assigns datasets by extracting or using keywords, however this solution will require more resources from data portal holders. As for visualisation and statistics, by which are meant visualization and analytical capabilities to obtain information about a dataset, such as charts or visualizations in maps, this is partly fulfilled - sometimes the possibility visualizing data is provided. As a result, the Latvian open data portal gains 16.7 out of 21 points for the "open dataset specification" while an average for $40+$ countries is 16.4 (see (Nikiforova, 2020b)).

For the second category, "open dataset feedback", which includes documentation and tutorials, forums and contact form etc., the Latvian open data portal scored 7.52 out of 12 points (an average for $40+$ countries is 7.68). Despite the fact that one of the frequently discussed challenges for open data portals and OGDs is that users receive very little feedback from the service providers (Smith et al., 2018), the Latvian open data portal provides an opportunity to contact the holder or publisher of each dataset. However, despite this option, it is not known whether users receive/ get feedback. This may be a research question for future studies. For the contact forms, a positive aspect is that contacting data publisher can be performed without logging or registering as expected. As for documentation and tutorials, a number of documents are provided, however, this aspect was not assessed by 3 points, as their quality and level of detail may not be sufficient for the normal user. The "user rating and comments" opportunity is not provided; however, it is possible to use "social media and sharing" opportunity. This aspect is perfectly realised by Cyprus, Ireland and Finland that can be considered as an example.

Regarding the "Request form", "List of requests", "Involvement in the process", the Latvia's Open Data Portal was assessed by 4.31 point out of 9 , while an average result is 5.16. This opportunity enables all stakeholders to request a new data set that could be of high interest to the public, but other stakeholders may vote in favour of this specific data set. This aspect allows to compare Latvia's Open Data Portal with Spain which portal is considered as one of the best portals, where this opportunity is provided as well, however, this showcase is even better since the number of possible values for the status of requests are provided such as assigned, published, not feasibly, under study, or partially published instead of published and not published as it is for Latvia. At the same time, Latvia's portal holders point out that this voting tool is of informative nature, so they have right to decide on the opening of these datasets by themselves that can be easily explained in several ways: (a) it is difficult to ensure the openness of all the data, in which the society may be interested in, because of challenges such as seeking an appropriate publisher, contacting him, preparing data sets etc., (b) users do not necessarily understand what the open data are, since a few requests were related to data that cannot be exploited as open data. Here the Ireland's portal should be mentioned, which supposes that the request can be commented, and as a result, the portal's employees explain to the users whether (and why) the request cannot be satisfied. 
Based on these results, it can be concluded that the "open dataset specification" for Latvia's open data portal is the best category, which gained a higher number of points (even better than an average for 40+ countries), however, the "open dataset feedback" got the worst. This result is also consistent with Open Data Maturity in Europe, according to which the impact of Latvia's portal is the worst. At the same time for OGD feedback is one of the crucial aspects of the impact criteria.

As for more specific aspects, "visualization and statistics", "user rating and comments" and "list of requests", appear to be the weakest points assessed by participants between "not fulfilled" and "partially fulfilled". These aspects must be improved as soon as possible, not only because of the low result but also because of the importance of these aspects. Moreover, it is also seen that these aspects are closely related to aspects that affect user experience, resulting in the impact of the open data initiative. It would also be recommended to address all aspects that have been assessed by less than 2.8 out of 3 points, however, these aspects are those which are already implemented in a relatively high way and are not such important until now. Nevertheless, they will be studied in the near future, with a detailed analysis and conclusion on how they could be improved. Some of them are also addressed in the next subsection.

Compared to another study carried out by the author of this study and her students, where 42 portals were evaluated, using the same methodology, it can be concluded that the Latvia's open data portal takes $16^{\text {th }}$ position (Nikiforova, 2020b). It demonstrates the competitiveness of the Latvia's open data portal at international level, but at the same time proves that there is room for improvements.

Although the methodology used is considered relatively objective and covers key aspects to be evaluated, further aspects need to be explored. These aspects were defined by the author as the user of this portal based on observations about using this.

\subsection{An Evaluation of the Portal from the Perspective of Experienced User}

This part of the study is based on the analysis of every data set currently available on Latvia's open data portal. Thus, 395 datasets were analysed from a variety of aspects including: (a) whether datasets are regularly updated; (b) whether the description of the parameters is provided (and what is its form); (c) whether API is supported; (d) whether a preview option is available.

One of the most crucial aspects for open data and OGDs, which affects users' intention to reuse data and increase their value, however, is rarely ensured, is a frequency of updates (Janssen et al., 2012), (Saxena, 2017), (Smith et al., 2018). Since there is a major challenge in most cases to understand when the data are up to date, this aspect was analysed taking into account the frequency of updates promised by the data publisher and the actual frequency of updates. First, data on the frequency of updates provided by data publishers were analysed. 13 different values for frequency of updates were identified, namely "daily", "weekly (once)", "monthly (once)", "constantly", "constantly updated", "quarterly", "annually (twice)", "annually (once)", "triennial", "irregular", "unknown", "never", "other". "Annually (once)" value appears to be the most popular value since it is valid for $27.3 \%$ of datasets, following by irregular, monthly, daily, annually (twice), never. Then, it was decided to examine whether the frequency of updates of the data was in line with real life. To perform this task, three parameters describing each dataset were analysed, namely "created", "last updated" and "frequency [of updates]" (defined by the data publisher). The results prove the necessity for such an analysis demonstrating that the value of "frequency" does not correspond to actual updates, since only $45.8 \%$ of data sets are updated as frequently as promised, $25.4 \%$ do not follow the promised frequency of updates, and for $28.8 \%$ data sets it is difficult to say at the moment since the value of "frequency" is greater than the difference between the publishing and the current dates. In addition, (a) the use of different names designating the same object or its property was observed for two values, namely "constantly" and "constantly updated" - it is recommended to involve a list of allowable values or to develop and introduce a vocabulary that would reduce this issue, (b) values such as "unknown" and "other" should not be allowed at all since they do not provide supposed information.

Another aspect that needs attention was a description of parameters aimed at clarifying, what the name of each parameter means. It was found that only $20 \%$ of datasets are supplied with a description of parameters, where the textual description appears to be less popular, since $54.4 \%$ of the data sets are supplemented with the description of the parameters that is available as a separate file. 
Another aspect related to the representation of data is whether the Application Programming Interface (API) is available. It is well known that data sharing in the form of machine-readable APIs is more useful for its further use (Sanderson et al., 2019) and is one of the most critical success factors for open data initiatives (Zuiderwijk et al., 2015). This is one of the most significant challenges for open data (McBride et al., 2018), and, in the context of Latvia, this is also valid, since only $43.04 \%$ of datasets (170 out of 395) are supplemented with API feature.

In addition, despite the fact that it is good practice to provide users with information on the number of applications (and preferably nature) based on open data that have been re-used - use-cases, which are able to attract more people to the portal and its data ((Zuiderwijk et al., 2015)), these data are not provided. One of such examples is demonstrated by our neighbours, the Lithuanian open data portal, which provides 9 use-cases of open data, and Poland (with 27 applications) providing them with both, links on the result and description. Of course, these two countries do not limit this list, since according to (Nikiforova, 2020b), the majority of countries provide this feature ( $71.43 \%$ of 42 analysed open data portals). In some cases, such as France, Luxembourg or Portugal, the national portal has even a "use case upload" feature, and 18 portals offer a mapping between the use cases and the datasets that the use cases are based on. One of such examples is Ireland. As for Latvia's portal, as a result of contacting representative organisations, it was found that such an information on hackathons is collected for internal use and a total of 29 applications were developed during two hackathons in 2018 and 2019. Most of them covers topics such as tourism, calculation queues to the hospitals or kindergarten, finding parking, finding the best place of life. Most of the developed services address topics that are crucial for Latvia and tries deciding sensitive problems that demonstrate the potential of open data and opportunities to improve citizens' lives. Therefore, it is strongly recommended to publish this information on the portal to demonstrate the positive effects of the use of open data, thereby increasing public participation. At the very early stage it would be recommended to follow at least Sweden example, where the list of "good examples" is provided with external list on the developed services.

However, although the abovementioned mainly concerns challenges, the current state of Latvia's open data portal is relatively high, that is proved by (Blank, 2019). Therefore, at least a few positive aspects should be mentioned in order to demonstrate why Latvia's open data portal holds sufficiently high positions in various ratings, despite a list of the challenges mentioned above that has not yet been solved. Among the positive aspects, some of which were already mentioned, there are: (a) the number of datasets and data publishers are provided and frequently updated; (b) two languages, namely Latvian and English, are supported; (c) the majority of datasets are downloadable and have at least basic set of [meta]data on it; (d) search and filter by category, data format, tags, data publisher; (e) a social media facility that can help create a social distribution channel for open data; (f) a request form (appears to be a problem to a list of countries) and (g) a contact facility with data publisher; (h) guidelines and news. However, aspects (e)-(g) have not been checked on whether they are working as intended, thus, it is difficult to draw any conclusions on their quality, and here only the fact of their presence is highlighted.

\section{CONCLUSION}

Nowadays, list of possible benefits and advantages motivate governments around the world to implement the OGD initiative, where the open data portal is one of the most important success factors. However, challenges and barriers raise major concerns about how to achieve successful implementation and results. If the quality of the open data portal is not high enough, potential data publishers may decide not to publish their data since they do not see a positive impact and their efforts will outweigh the positive effects they may have, but data users will not trust the data and will not find this portal usable and fit for their purposes. This study is of high importance, as (a) critical voices on the OGD and the open data portal appear in Latvia, (b) a literature review demonstrates that there are no studies focused on the OGD initiative, and, in particular, open data portal of Latvia. This is a gap that is covered by this paper, since the current situation is being assessed, and corrective actions are being recommended, which will hopefully move towards the effective implementation of the open data portal in Latvia.

The proposed study seeks to address the various researchers' calls by dividing the analysis into three parts: (a) a survey which studies the knowledge of the Latvian society, (b) the application of (Máchová et al., 2018) framework to the Latvia's open data portal, (c) an analysis of additional aspects inspecting each data set. The implementation of the guidelines defined should also improve user participation by encouraging the 
awareness and reuse of open data, which appears to be one of the most important aspects for each country opening its data, and that is one of the weakest points for Latvia, since the Latvian open data portal is certainly usable as assessed by the participants (6.3 out of 10 points) and demonstrated by the application of framework, however, there is room for improvements listed in this paper.

Although the results of this study are more important mainly for open data portal holders, they could also be useful for users, as these results (1) point to the weakest points to be taken into account using open data portal and (b) provide aspects to be considered when selecting the portal to be used. In addition, potential open data publishers can use the results provided as a checklist when preparing their data for publishing.

This study will be continued to carry out an in-depth analysis of the identified challenges and to propose guidelines to address them, as well as to provide a detailed comparison with more successful open data portals. A detailed literature review will also follow.

\section{ACKNOWLEDGMENT}

This work has been supported by University of Latvia Faculty of Computing project AAP2016/B032 "Innovative information technologies". I am also grateful to the participants taking part in the experiment within my workshop "Open data and data quality", which allowed to collect data and led to such results, and Latvian Open Technology Association for sharing information regarding open data hackathons.

\section{REFERENCES}

Andrea, P., Alberto, P., \& Barbara, R. (2012). E-government Services and User Expectations. In Proceedings of the International Conference on e-Learning, e-Business, Enterprise Information Systems, and e-Government (EEE) (p. 1). The World Congress in Computer Science, Computer Engineering and Applied Computing (WorldComp).

Attard, J., Orlandi, F., Scerri, S., Auer, S. (2015). A systematic review of open government data initiatives. Government Information Quarterly, 32(4), 399-418.

Blank, M. (2019) Open Data Maturity Report 2019, European Data Portal, online: https://www.europeandataportal.eu/sites/default/files/open_data_maturity_report_2019.pdf

Bojars, U., \& Liepins, R. (2014). The State of Open Data in Latvia: 2014. Baltic Journal of Modern Computing, pp.160.

Faini, F., \& Palmirani, M. (2016). Italian Open and Big Data Strategy. In International Conference on Electronic Government and the Information Systems Perspective (pp. 105-120). Springer, Cham.

Janssen, M., Charalabidis, Y., Zuiderwijk, A. (2012). Benefits, adoption barriers and myths of open data and open government. Information systems management, 29(4), 258-268.

Kim, H. (2019). Analysis of standard vocabulary use of the open government data: the case of the public data portal of Korea. Quality \& Quantity, 53(3), 1611-1622.

Máchová, R., Hub, M., Lnenicka, M. (2018). Usability evaluation of open data portals. Aslib Journal of Information Management.

McBride, K., Toots, M., Kalvet, T., Krimmer, R. (2018). Open Government Data Driven Co-creation: Moving Towards Citizen-Government Collaboration. In International Conference on Electronic Government (pp. 184-195). Springer.

Nikiforova, A. (2020a) Definition and Evaluation of Data Quality: User-Oriented Data Object-Driven Approach to Data Quality Assessment. Baltic Journal of Modern Computing, 8(3), 391-432.

Nikiforova, A. (2020b) Comparative Analysis of the usability of National Open Data Portals or Whether Your Open Data Portal is Ready to Bring Benefits from Open Data (in print).

Nikiforova, A. (2019). Analysis of Open Health Data Quality Using Data Object-Driven Approach to Data Quality Evaluation: Insights from a Latvian Context, In IADIS International Conference e-Health 2019, Part of the IADIS Multi Conference on Computer Science and Information Systems, pp.119-126, MCCSIS 2019. IADIS.

Sanderson, T., Reeson, A., Box, P. (2019). Optimizing Open Government: an economic perspective on data sharing. In Proceedings of the 12th International Conference on Theory and Practice of Electronic Governance (pp. 140-143).

Saxena, S. (2017). "Usage by stakeholders" as the objective of "transparency-by-design" in open government data. Information and Learning Science.

Smith, G., Sandberg, J. (2018). Barriers to innovating with open government data: Exploring experiences across service phases and user types. Information Polity, 23(3), 249-265.

Zuiderwijk, A., Susha, I., Charalabidis, Y., Parycek, P., Janssen, M. (2015). Open data disclosure and use: Critical factors from a case study. In CeDEM15: Conference for E-Democracy and Open Government (Vol. 197). MV-Verlag. 